\title{
Correction to: Genomic comparisons of Streptococcus suis serotype 9 strains recovered from diseased pigs in Spain and Canada
}

Han Zheng ${ }^{1 \dagger}$, Pengcheng Du ${ }^{2 \dagger}$, Xiaotong Qiu ${ }^{1}$, Anusak Kerdsin ${ }^{3}$, David Roy ${ }^{4}, X_{\text {Xuemei Bai }}{ }^{1}$, Jianguo Xu', Ana I. Vela ${ }^{5}$ and Marcelo Gottschalk ${ }^{4^{*}}$ (i)

\section{Correction to: Vet Res 49:1 (2018)}

https://doi.org/10.1186/s13567-017-0498-2

In the original publication of this article [1], the author name 'Pengchen Du' in author list should be 'Pengcheng Du'.

\section{Author details}

${ }^{1}$ State Key Laboratory of Infectious Disease Prevention and Control, Collaborative Innovation Center for Diagnosis and Treatment of Infectious Diseases, National Institute for Communicable Disease Control and Prevention, Chinese Center for Disease Control and Prevention, Changping, Beijing, China. ${ }^{2}$ Beijing Key Laboratory of Emerging Infectious Diseases, Institute of Infectious Diseases, Beijing Ditan Hospital, Capital Medical University, Beijing, China.

${ }^{3}$ Faculty of Public Health, Kasetsart University Chalermphrakiat Sakon Nakhon Province Campus, Bangkok, Sakon Nakhon, Thailand. ${ }^{4}$ Swine and Poultry Infectious Diseases Research Center, Faculty of Veterinary Medicine, University of Montreal, Montreal, QC, Canada. ${ }^{5}$ Departamento de Sanidad Animal,

Facultad de Veterinaria and Centro de Vigilancia Sanitaria Veterinaria (VISAVET), Universidad Complutense de Madrid, Madrid, Spain.

The original article can be found online at https://doi.org/10.1186/s1356 7-017-0498-2.

\section{Reference}

1. Zheng H, Du P, Qiu X, Kerdsin A, Roy D, Bai X, Xu J, Vela Al, Gottschalk M (2018) Genomic comparisons of Streptococcus suis serotype 9 strains recovered from diseased pigs in Spain and Canada. Vet Res 49:1. https:// doi.org/10.1186/s13567-017-0498-2

\section{Publisher's Note}

Springer Nature remains neutral with regard to jurisdictional claims in published maps and institutional affiliations.

Published online: 16 September 2019

\footnotetext{
*Correspondence: marcelo.gottschalk@umontreal.ca

${ }^{\dagger}$ Han Zheng and Pengcheng Du contributed equally to this work

${ }^{4}$ Swine and Poultry Infectious Diseases Research Center, Faculty

of Veterinary Medicine, University of Montreal, Montreal, QC, Canada

Full list of author information is available at the end of the article
} 